\title{
Unconventional ordering behavior of semi-flexible polymers in dense brushes under compression
}

\author{
Andrey Milchev ${ }^{1,2}$ and Kurt Binder ${ }^{2}$ \\ 1 Institute of Physical Chemistry, Bulgarian Academy of Sciences, Sofia 1113, Bulgaria \\ ${ }^{2}$ Institut für Physik, Johannes Gutenberg-Universität Mainz, Staudinger Weg 7, D-55099 Mainz, Germany
}

\begin{abstract}
Using a coarse-grained bead-spring model for semi-flexible macromolecules forming a polymer brush, structure and dynamics of the polymers is investigated, varying chain stiffness and grafting density. The anchoring condition for the grafted chains is chosen such that their first bonds are oriented along the normal to the substrate plane.

Compression of such a semi-flexible brush by a planar piston is observed to be a two-stage process: for small compressions the chains contract by "buckling" deformation whereas for larger compression the chains exhibit a collective (almost uniform) bending deformation. Thus, the stiff polymer brush undergoes a 2-nd order phase transition of collective bond reorientation. The pressure, required to keep the stiff brush at a given degree of compression, is thereby significantly smaller than for an otherwise identical brush made of entirely flexible polymer chains! While both the brush height and the chain linear dimension in the $z$-direction perpendicular to the substrate increase monotonically with increasing chain stiffness, lateral $(x y)$ chain linear dimensions exhibit a maximum at intermediate chain stiffness. Increasing the grafting density leads to a strong decrease of these lateral dimensions, compatible with an exponential decay. Also the recovery kinetics after removal of the compressing piston is studied, and found to follow a power-law / exponential decay with time.

A simple mean-field theoretical consideration, accounting for the buckling/bending behavior of semi-flexible polymer brushes under compression, is suggested.
\end{abstract}

\section{INTRODUCTION}

Since several decades polymer brushes find abiding interest for various applications, and have been studied very intensively by experiment, analytical theory, and computer simulations [1 11]. The structure of these soft polymeric layers and their response to various external perturbations depends in a delicate way on various control parameters such as molecular weight, grafting density, quality of the solvent, and character of the interactions between monomeric units and the substrate to which the macromolecules are grafted. However, inspired by the early works [12, 13], mostly brushes formed from completely flexible chains were considered, where the elastic response is entirely entropic, due to the configurational degrees of freedom of the tethered chain molecules. In such brushes, chains may be nevertheless strongly stretched in the direction perpendicular to the substrate, to avoid unfavorable monomer-monomer overlap in the case of sufficiently dense grafting.

Effects of intrinsic chain stiffness, as are expected for semi-flexible polymers [14], did find rather little attention [15 19], apart from the case where one considers grafted semi-flexible chains in nematic solvents [20, 21] and their liquid crystalline order [22, 23]. We also draw attention to some related problems where rod-like molecules densely packed and anchoring at substrates play a role, such as in Langmuir monolayers of surfactants and related systems 24 27]. Experimentally, the interactions between DNA-grafted colloids [28, 29], or poly(acrylic acid) brushes with variable rigidity [30], have been comprehensively investigated by F. Kremer and collaborators. In recent years, semiflexible polymers find increasing interest particularly in the context of biophysical systems, such as cytoskeleton actin bundles [31 35] biofilaments [36 38], and also biobased polymer brushes were recently created [39]. Thus, a more comprehensive simulation study of brushes formed from semi-flexible polymers would be desirable to theoretically clarify the effect of chain stiffness on structure and dynamics of such systems, including also their response to external perturbations, such as shear and compressive forces.

As is well known, one important possible application of polymer brushes formed from flexible polymers is their use as a lubricant [4, 6, 10, 40 42], although the theoretical aspects of this response to shear and compression are still under discussion [43 48]. In our preliminary publication [49], indeed, an anomalous response of brushes formed from rather stiff chains was found, caused by an onset of orientational order parallel to the substrate.

In the present paper we shall give a more complete account of this problem, focusing mainly on the structure and static behavior of such stiff brushes subject to compression. In the next Section [1 the model that is studied will be introduced and the simulation method will be described. Section III examines the parallel and perpendicular chain linear dimensions in rigid brushes as a function of bending rigidity and grafting density over a wide range, as well as the profiles of monomer density in the direction perpendicular to the grafting substrate, and the end monomer distribution. Then in Section IV we elaborate the topic that was already briefly addressed in our preliminary study, compression of such brushes by a piston, presenting new results, including also the variation with grafting density and chain length. The hysteresis associated with the transition towards orientational order will also be investigated. 
Section $\mathrm{V}$ is devoted to an attempt for theoretical consideration within the Mean-Field Approximation of the brush response to deformation. A brief account on the recovery dynamics of compressed stiff brushes is then given in Section VI. Our conclusions are summarized in section VII.

\section{MODEL AND SIMULATION METHODS}

We start from the standard model for polymer brushes formed from completely flexible polymers [3, 50] and complement it by adding a bond angle potential. This standard model is a bead-spring model in the continuum, where bonded effective monomers interact with the well-known finitely extensible non-linear elastic (FENE) potential [51]

$$
V^{F E N E}(r)=-0.5 k r_{0}^{2} \ln \left[1-\left(r / r_{o}\right)^{2}\right], 0<r<r_{o}
$$

where $r$ is the distance between the beads, and the spring constant $k$ as well as the maximum distance $r_{0}$ between neighboring monomers will be specified below. In addition, for any pairs of monomers (both bonded and non-bonded ones) a truncated and shifted Lennard-Jones potential acts, the so-called Weeks-Chandler-Andersen (WCA) potential $52]$

$$
V^{W C A}(r)=4 \epsilon\left[(\sigma / r)^{12}-(\sigma / r)^{6}+1 / 4\right], r<r_{c}=2^{1 / 6} \sigma
$$

while $V^{W C A}\left(r>r_{c}\right)=0$. Note that this potential is cut off in its minimum and both potential and forces are continuous for $r=r_{c}$. We henceforth take $\sigma=1$ as our unit of length, and $\epsilon=T=1$ (also Boltzmann's constant $k_{B}$ is taken as unity, as usual). No explicit solvent molecules are included, Eqs. 1, 2] describe interactions between effective monomers and solvent molecules implicitly only, corresponding to the very good solvent regime. The constants of the FENE potential are chosen as

$$
r_{0}=1.5 \sigma, \quad k=30 \epsilon / \sigma^{2}
$$

so that the total potential between two subsequent monomers along the chain $\left(V^{F E N E}(r)+V^{W C A}(r)\right)$ has a minimum at about $r_{\min }=0.96 \sigma$.

The flexibility of the chains now is varied by introducing a bond angle potential $V_{b}\left(\vartheta_{i j k}\right)$ that depends on the angle $\vartheta_{i j k}$ between the bonds between monomer pairs $(i, j)$ and $(j, k)$ respectively

$$
V_{b}\left(\vartheta_{i j k}\right)=\kappa_{b}\left[1-\cos \left(\vartheta_{i j k}\right)\right]
$$

For rather stiff chains we have $\kappa_{b} \gg 1$ and hence Eq. 4 can be approximated as

$$
V_{b}\left(\vartheta_{i j k}\right) \approx \frac{\kappa_{b}}{2} \vartheta_{i j k}^{2}
$$

which shows that our polymer model can be viewed as a discretized version of the well-known Kratky-Porod model [53] of semiflexible chains (but, unlike the Kratky-Porod model, the present model fully accounts for excluded volume effects and is hence suitable for the study of rather dense systems as well). Note that the effective persistence length of an isolated chain would be $\ell_{p}=\kappa_{b} \sigma / k_{B} T=\kappa_{b}$; of course. Due to packing effects in dense systems, local nematic order may arise as a result of interchain interactions that lead to nematic short or long range order, and then the persistence length can be appreciably larger (see e.g. [54]). For small $\kappa_{b}$, the persistence length is, in general, not a well defined quantity [55].

The chains are grafted at a planar $L \times L$ impenetrable surface, choosing a square lattice arrangement of grafting sites, the lattice spacing chosen according to the desired grafting density $\sigma_{g}$. Alternatively, also a random arrangement of grafting sites has been considered, as discussed below. The substrate surface exerts a repulsive interaction of a form analogous to Eq. 3 on all monomers,

$$
V^{\text {wall }}(z)=4 \epsilon_{w}\left[\left(\sigma_{w} / z\right)^{12}-\left(\sigma_{w} / z\right)^{6}+\frac{1}{4}\right], \quad 0<z<z_{c}=\sigma_{w} 2^{1 / 6} .
$$

Here $z$ is the distance of a monomer from the wall, and the parameters are chosen as $\epsilon_{w}=\epsilon$, $\sigma_{w}=\sigma$. In $x y$-directions, periodic boundary conditions are used, and in $z$-direction the simulation box is closed with another repulsive wall (at distance $D$ from the grafting surface) where a potential of the same type as Eq. 6 acts (simply $z$ needs to be replaced by $D-z$ on the right hand side of Eq. 6). This upper wall has no physical effect, as long as $D$ exceeds significantly the height, $h_{0}$, of the unperturbed brush in equilibrium. However, we shall consider also the effect of compressing the brush by reducing $D$ to values $D<h_{0}$. 
As an initial condition, all chains are put into a straight rod configuration, choosing all angles $\theta_{i j k}=0$. Note that the first bond is constrained to the $z$-direction, perpendicular to the grafting surface [66], so in the initial state all chains are oriented along the $z$-axis. Then the chain configurations are relaxed by standard Molecular Dynamics (MD) simulations, applying the Velocity Verlet algorithm [56] and a Langevin thermostat. Thus the coordinates $\vec{r}_{i}(t)$ of the effective monomers evolve according to the Newton equations of motion

$$
m \frac{d^{2} \vec{r}_{i}}{d t^{2}}=-\frac{\partial V^{t o t}\left(\left\{\vec{r}_{j}\right\}\right)}{\partial \vec{r}_{i}}-\gamma \frac{d \vec{r}_{i}}{d t}+\vec{F}_{i}(t)
$$

where the mass of effective monomers $m$ is chosen to be unity as well, $V^{\text {tot }}$ is the total potential (containing the terms from the interactions between monomers, Eqs. 1 2, 4 and the wall-monomer interaction, Eq,6), $\gamma$ is a friction coefficient and $\vec{R}_{n}(t)$ a random stochastic force. The latter satisfies the fluctuation-dissipation relation [56, 57] $(\alpha, \beta$ denote the Cartesian coordinates)

$$
\left\langle F_{i}^{\alpha}(t) F_{j}^{\beta}\left(t^{\prime}\right)\right\rangle \equiv 2 k_{B} T \gamma \delta_{i j} \delta_{\alpha \beta} \delta\left(t-t^{\prime}\right)
$$

The friction coefficient $\gamma$ is chosen as $\gamma=0.25$. This choice ensures efficient equilibration. We also note that with our choice of units, the MD time unit is unity as well, $\tau_{M D}=\sigma(m / \epsilon)^{1 / 2}=1$. In our simulations, $\kappa_{b}$ is varied from $\kappa_{b}=0$ (flexible chains) up to $\kappa_{b}=50$, and chain lengths from $N=10$ to $N=80$, so cases are included where the contour length and the persistence length are of the same order of magnitude. Grafting densities are varied from $\sigma_{g}=0.0625$ (the "mushroom regime" [11 13] ) to $\sigma_{g}=1$ (in the latter case monomer density inside of the brush corresponds to a concentrated solution). The number of chains was typically chosen to be $\mathcal{N}_{\text {chain }}=128$, but in a few cases the lateral box linear dimensions were doubled (so that $\mathcal{N}_{\text {chain }}=512$ ) so as to verify that finite size effects are still negligibly small.
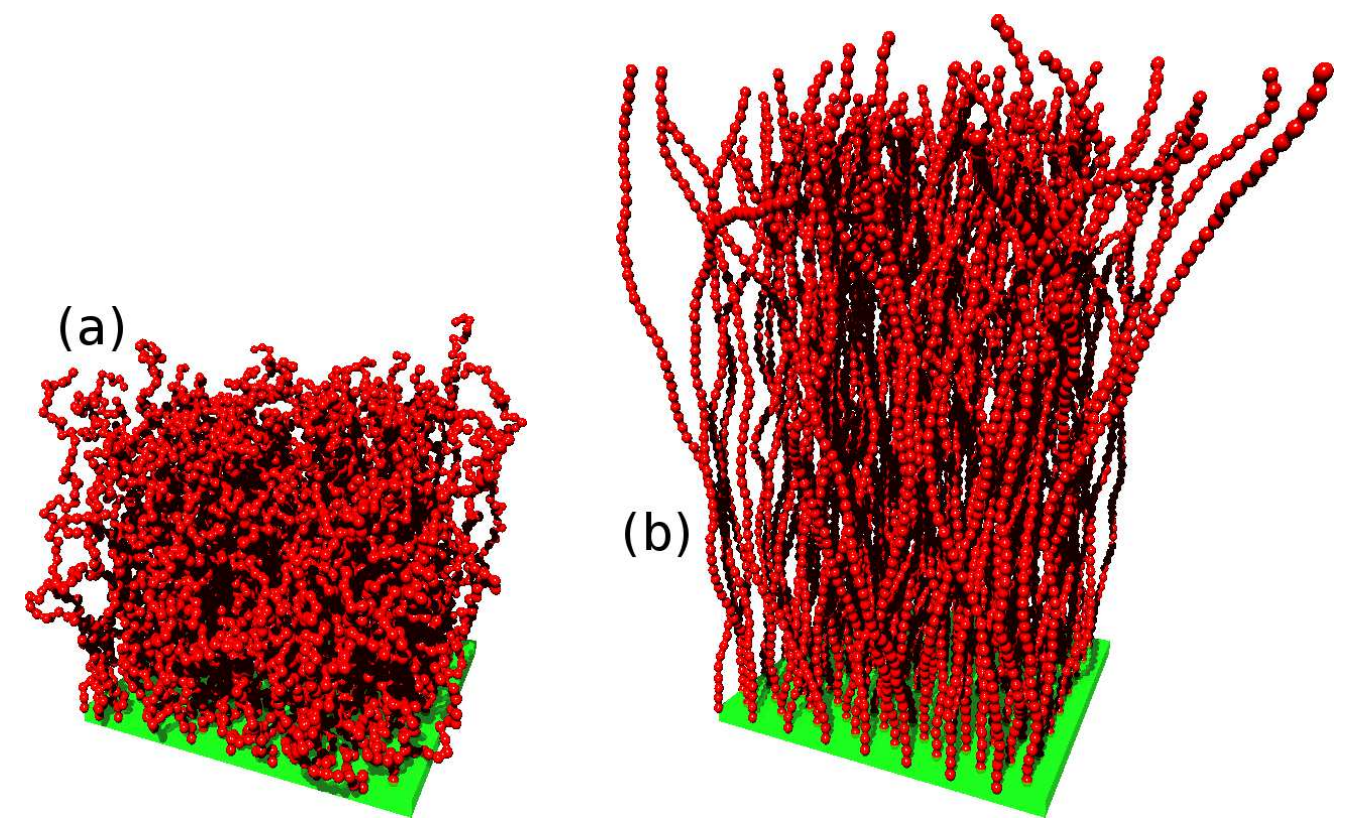

FIG. 1: Snapshot pictures of polymer brushes for the case $L=32, \sigma_{g}=0.125, N=60$, and two choices of $\kappa_{b}$ : $\quad \kappa_{b}=0$ (fully flexible chains) (a), $\kappa_{b}=50$ (semi-rigid chains) (b). The distance between observer and objects is the same in both cases.

As an example, Fig. 1 1 shows typical snapshot pictures for a well-equilibrated brush at a grafting density $\sigma_{g}=0.125$ and $N=60$ for two choices of $\kappa_{b}, \kappa_{b}=0,50$. One can clearly see that for the flexible case the chain conformation is locally rather random, the overall stretching of the chains in $z$-direction becomes visible on larger scales only. In contrast, the stiff chains show at most long wavelength transverse undulations, like a bunch of pliant rods. This behavior is exactly what one expects to occur for worm-like chains, which hence are well modeled by our simulation. In the next section we shall analyze the properties of these brushes in more detail. 


\section{STRUCTURE OF SEMIFLEXIBLE POLYMER BRUSHES}

We start with a discussion of the monomer density profile, $\phi(z)$, and the distribution of free ends, $\rho(z)$, for a typical choice of $N$ and $\sigma_{g}, N=40$ and $\sigma_{g}=0.25$, varying $\kappa_{b}$ (Fig. 2a ).

One notes that near the wall there is always a pronounced layering effect, which strongly increases with $\kappa_{b}$, although the density of the stiffer chains is smaller in the center of the brush (near $z=h / 2$, as a rough measure of brush height $h$, we may take the inflection point of the profile $\phi(z)$ near its final decay for large $z$ ). This density decrease with increasing $\kappa_{b}$ must occur since the stiffer chains are more stretched and the brush height $h_{0}$ increases with increasing $\kappa_{b}$ up to its maximum value while the number of monomers stays constant. This effect that stiffer chains exhibit more pronounced layering than flexible chains has already been pointed out for a different model [19]. Note that the actual value of the density is $N \phi(z)$ and hence about 0.44 for $\kappa_{b}=0,0.33$ for $\kappa_{b}=5$, and 0.26 for $\kappa_{b}=50$. These densities are much smaller than melt densities, indicating a rather unexpected high degree of order for the semi-flexible brushes near the wall. Fig. $2 \mathrm{~b}$ shows that the distribution of chain ends becomes sharper with increasing chain stiffness. The half-width $\delta h_{1 / 2}$ scales as $\delta h_{1 / 2} \propto \kappa_{b}^{-0.5}$ for large $\kappa_{b}$ (i.e., $\left(\delta h_{1 / 2}\right)^{2}$ scales inversely with the persistent length).
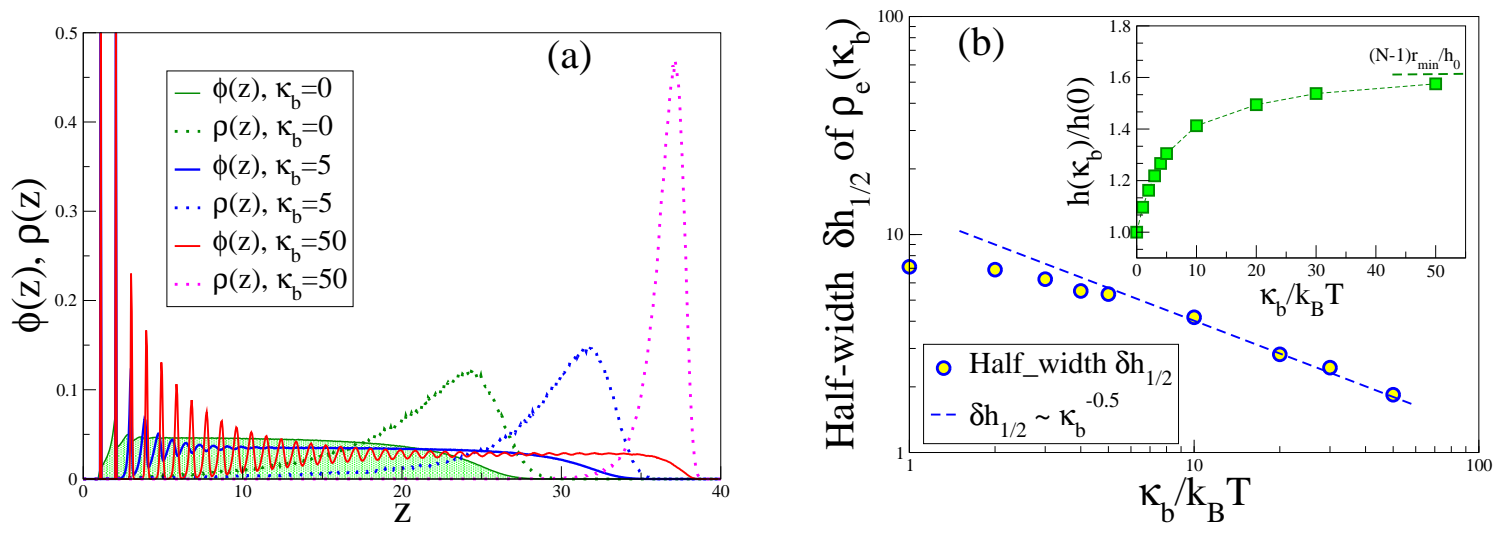

FIG. 2: (a) Density distribution $\phi(z)$ of the effective monomers in the brush, and distribution of chain ends, $\rho(z)$, plotted vs. the distance $z$ from the grafting surface, for $N=40, \sigma_{g}=0.25$, and three choices of $\kappa_{b}$, as indicated. The density profile for $\kappa_{b}=0$ is indicated by shaded area. Note the normalizations $\int_{0}^{\infty} \phi(z) d z=1, \int_{0}^{\infty} \rho(z) d z=1$. (b) Half-width of $\rho\left(\kappa_{b}\right)$ vs $\kappa$ for $N=40$ and $\sigma_{g}=0.25$, shown as a log-log plot. The inset shows the relative increase of the brush height with $\kappa_{b}$. The asymptotic value $h\left(\kappa_{b} \rightarrow \infty\right) / h_{0}=(N-1) r_{\text {min }} / h_{0}$ is indicated by the dashed horizontal line.

The height of the brush $h\left(\kappa_{b}\right)$ saturates for $\kappa_{b} \rightarrow \infty$ at its maximum value of fully stretched chains, $h_{\max }=$ $0.96(N-1)$, see the inset of Fig. 2 2 b, so the distribution of chain ends becomes a delta-function! Fig. 3 presents the chain linear dimensions as functions of $\kappa_{b}$, for $N=40$ and $\sigma_{g}=0.25$. As expected, the chains get more stretched as the polymers get stiffer. Interestingly, also the linear dimensions in $x y$-directions parallel to the wall initially increase even stronger than the linear dimensions in $z$-direction. Due to the increased stretching, the density in the brush has decreased, and hence there is more space for the monomers to occupy. In particular, the large magnitude of this effect is surprising. It is also evident from the snapshot - Fig. 1 $\mathrm{b}$. We also note that the linear dimensions $R_{g x y}^{2}, R_{x y}^{2}$ reach their maximum in the range $10 \leq \kappa_{b} \leq 20$, i.e., a range where the contour length is just a few times larger than the persistence length. Note that for a single semi-flexible chain one predicts that transverse fluctuations of monomers in the rod-like limit scale as [58]

$$
\left\langle\left(\delta \vec{r}_{x y}\right)^{2}\right\rangle \propto L^{3} / \ell_{p}
$$

Thus, Fig. 3 suggests that a similar decrease of the linear dimensions in the $x y$-direction (transverse to the average orientation of the rod-like grafted chains in the brush, which is the $z$-direction) occurs also in fairly dense brushes. However, much more data (for still larger values of $\kappa_{b}$ ) would be required to test whether Eq. 9 holds here quantitatively. Note that for $\kappa_{b}=50$ the $x y$-components of the mean square gyration radius are still larger than their counterparts for flexible chains, although the brush height is close to its maximum value. For $\kappa_{b} \rightarrow \infty$, these $x y$-components must tend to zero.

Fig. [4studies the variations of chain linear dimensions with $\sigma_{g}$. While for flexible chains one knows that in the semidilute limit $R_{z} \propto \sigma_{g}^{1 / 3} N$, and hence $R_{g z}^{2} \propto \sigma_{g}^{2 / 3} N^{2}$, that is, our data are compatible with a variation $R_{g z}^{2} \propto N^{2}$, but 


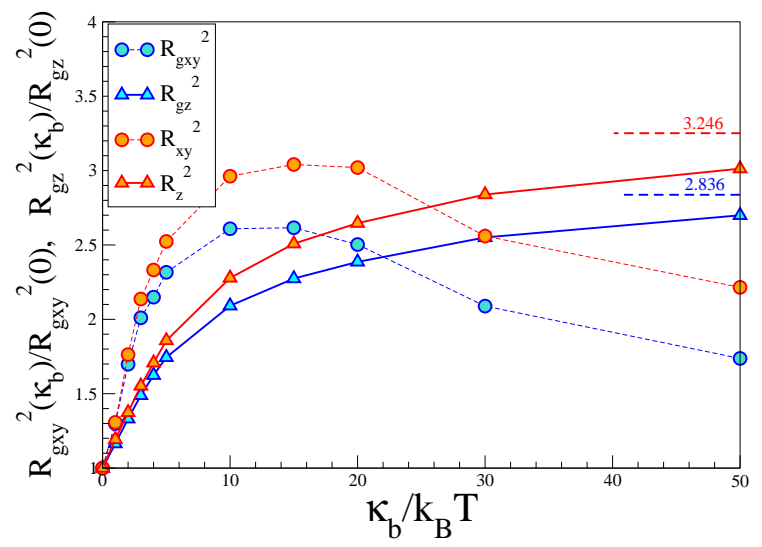

FIG. 3: Normalized mean square gyration radii components and end-to-end distance components $R_{g z}^{2}\left(\kappa_{b}\right) / R_{g z}^{2}(0), \quad R_{z}^{2}\left(\kappa_{b}\right) / R_{z}^{2}(0), R_{g x y}^{2}(\kappa) / R_{g x y}^{2}(0), \quad R_{x y}^{2}\left(\kappa_{b}\right) / R_{x y}^{2}(0)$ in the directions perpendicular $(z)$ and parallel $(x y)$ to the grafting surface, plotted vs. $\kappa_{b}$ for $N=40$ and $\sigma_{g}=0.25$. Dashed horizontal lines indicate the asymptotic ratio values for $\kappa_{b} \rightarrow \infty$ whereby $R_{z}\left(\kappa_{b} \rightarrow \infty\right)=(N-1) r_{\min }$ and $R_{g z}\left(\kappa_{b} \rightarrow \infty\right)=\frac{1}{12}\left[r_{\min }(N-1)\right]^{2}$.

the variation with $\sigma_{g}$ is distinctly weaker. The values reached for these large values of $\sigma_{g}$ indicate that the chains are stretched out like straight rods, since in this rod limit we simply have $R_{z}^{2} / N^{2} \approx 0.92$ and $R_{g z}^{2} / N^{2}=r_{\min }^{2} / 12=0.077$, respectively. Note that for $\sigma_{g} \geq 0.5$ thus a saturation is reached.
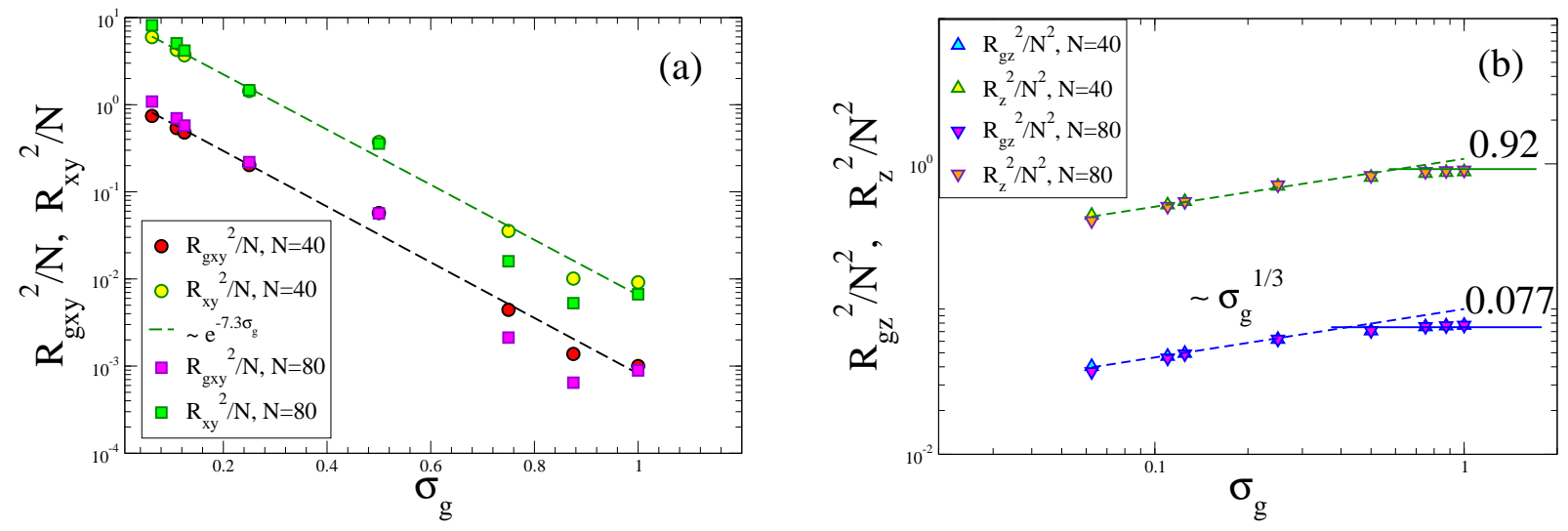

FIG. 4: (a) Plot of $R_{g x y}^{2} / N$ and $R_{x y}^{2} / N$ vs. $\sigma_{g}$ for $\kappa_{b}=20$ and two choices of $\mathrm{N}, \mathrm{N}=40$ and $\mathrm{N}=80$. Note the logarithmic ordinate scale; the straight lines indicate an empirical exponential relation, $\exp \left(-7.3 \sigma_{g}\right)$. (b) $\log$ - $\log$ plot of $R_{g z}^{2} / N^{2}$ and $R_{z}^{2} / N^{2}$ vs. $\sigma_{g}$. Straight lines indicate variations proportional to $\sigma_{g}^{1 / 3}$. All data refer to $\kappa_{b}=20$. Both data for $N=40$ and $N=80$ are shown. Horizontal straight lines in (b) indicate the asymptotic results when the chains are fully stretched out like a straight rod along the $z$-axis, i.e., $R_{z}^{2} / N^{2}=r_{\min }^{2} \approx 0.92$ and $R_{g z}^{2} / N^{2}=r_{m i n}^{2} / 12=0.077$, respectively.

Similarly, for flexible chains one would have $R_{g x y}^{2} \propto \sigma_{g}^{-1 / 6} N$; however, the present data suggest a much more rapid decay of $R_{g x y}^{2}$ with $\sigma_{g}$ than this weak power law. Empirically we find that the decrease is compatible with an exponential decay: Unfortunately, we do not have any explanation for this puzzling behavior. However, it should be remarked that for semi-flexible brushes the regime where the crossover from semi-dilute brushes to mushroom-like behavior occurs is found for much smaller grafting densities than for brushes formed from flexible chains of the same length. This fact simply results from the observation that the size of a chain in dilute solution scales as $R \propto \ell_{b} N^{3 / 5}$ for flexible chains, while for semi-flexible chains the size is much larger [59], $R \propto \ell_{p}^{1 / 5} \ell_{b}^{4 / 5} N^{3 / 5}$ for very long chains $\left(N>\left(\ell_{p} / \ell_{b}\right)^{3}\right)$ and $R \propto\left(\ell_{p} \ell_{b}\right)^{1 / 2} N^{1 / 2}$ for chains with $\ell_{p} / \ell_{b}<N<\left(\ell_{p} / \ell_{b}\right)^{3}$; the bond length is denoted as $\ell_{b}$ in this scaling description, and can be identified with the distance $r_{\min }$ of the minimum of our effective potential between neighboring monomers in our model. Since we use here rather stiff chains of medium length, we are in the regime 

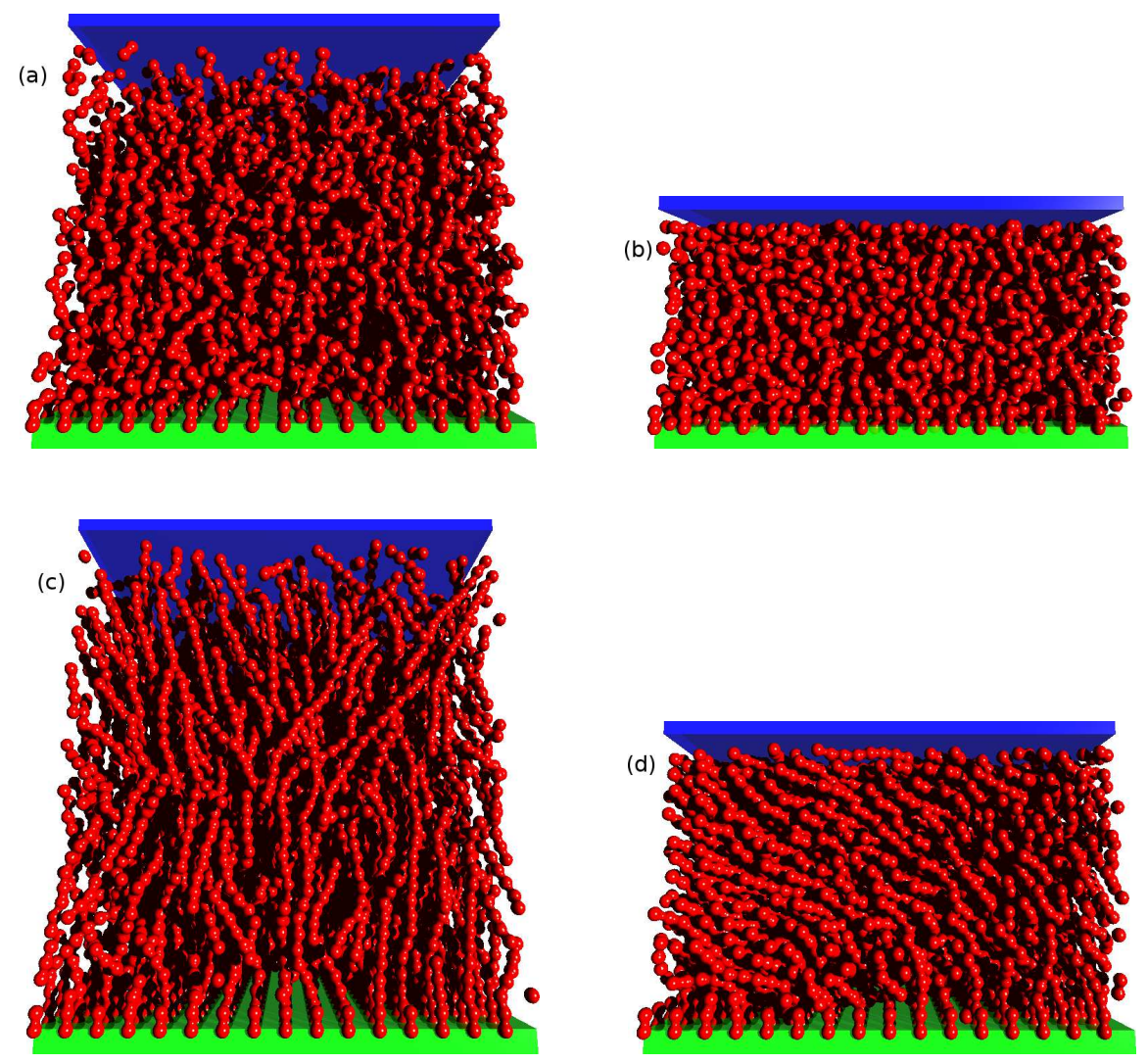

FIG. 5: Snapshot pictures of polymer brushes for the case of $\mathrm{L}=32, \mathrm{~N}=40, \sigma_{g}=0.25$, and two choices of $\kappa_{b}, \kappa_{b}=0(\mathrm{a}, \mathrm{b})$ and $\kappa_{b}=20(\mathrm{c}, \mathrm{d})$, applying a compression characterized by $D / h_{0}=0.9(\mathrm{a}, \mathrm{c})$ and $D / h_{0}=0.5(\mathrm{~b}, \mathrm{~d})$.

$N<\left(\ell_{p} / \ell_{b}\right)^{3}$ with our simulations always. Hence the crossover from mushrooms to semi-dilute brushes occurs for $\sigma_{g}=\sigma_{g}^{*}$ given by

$$
\sigma_{g}^{*} \approx R^{-2}=\ell_{p}^{-1} \ell_{b}^{-1} N^{-1}
$$

The scaling behavior typical for semi-flexible semi-dilute brushes hence is expected for $\sigma_{g}^{*} \ll \sigma_{g} \ll 100 \sigma_{g}^{*}$. This regime clearly is not explored in our simulations, which rather address the regime from concentrated solutions to melt densities.

\section{RESPONSE OF SEMIFLEXIBLE BRUSHES TO COMPRESSION - SIMULATION RESULTS}

In this section we consider the change of the state of the brush when it is compressed by a flat structureless piston parallel to the grafting surface that is brought to a height $D$ (= distance from the grafting surface) less than the height $h_{0}$ of the free brush. Fig. 5 shows a few representative snapshot pictures and Fig. 6 corresponding density profiles. One can see that for the flexible chain the density increase caused by the compression leads to a more pronounced layering of the monomers near the grafting surface; also at the compressing upper surface the parabolic decay of the density profile found in the free brush is replaced by the density oscillations in the compressed brush. For very strong compression the layering at the compressing wall is even more pronounced than near the grafting surface, where the brush conformation is more constrained due to the condition that the first bond of each grafted chain must be oriented in the $z$-direction perpendicular to the grafting surface. For the semi-flexible grafted chains the compression has the effect to clearly reduce the extent in $z$-direction over which layering can be seen, in comparison with the corresponding uncompressed brush.

The snapshot pictures (Fig. (5) give a qualitative interpretation for this surprising behavior: While for the flexible brush the chains get uniformly compressed and the density increases gradually, the rather stiff chains exhibit a 

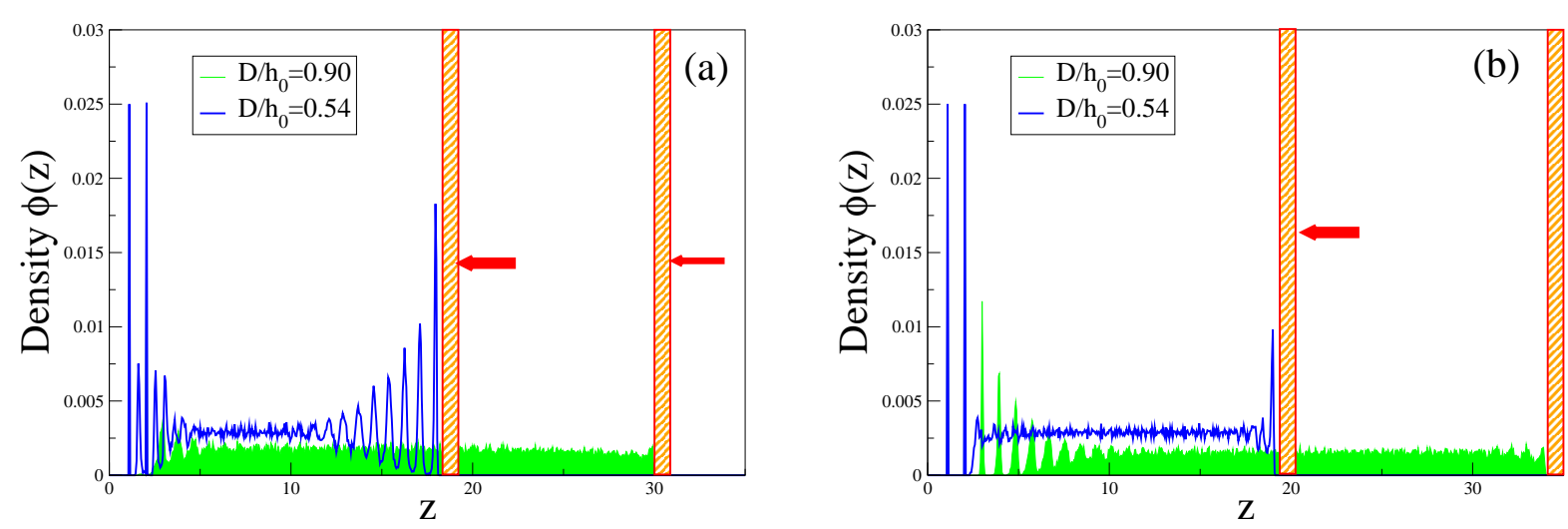

FIG. 6: Density distribution of the effective monomers in the brush, $\phi(z)$, plotted vs distance $z$ from the grafting surface, for $N=40, \sigma=0.5$, for two choices of $D / h_{0}$, namely, $D / h_{0}=0.9$ and $D / h_{0}=0.54$. Two choices of $\kappa_{b}$ are included namely fully flexible chains $\left(\kappa_{b}=0\right.$, case $(\mathrm{a})$, and semiflexible ones $\left(\kappa_{b}=20\right)$, case $(\mathrm{b})$.

collective bending, and the interplay of the bending of the stiff chains and the dense packing of the effective monomers destroys the periodicity of the local density that we see in the uncompressed case. It is found that the onset of collective chain bending does not start immediately when the distance $D$ between the compressing piston surface and the grafting substrate become equal to the uncompressed brush height $h_{0}$, but only when the degree of compression $1-D / h_{0}$ exceeds a threshold value. This fact is clearly recognized (Fig. (7) when we study the normalized pressure $\sigma P / k_{B} T$ (note that the normal pressure $P$, i.e. the diagonal component of the pressure tensor $p_{z z}$, is easily sampled using the virial theorem [56]). For a free, uncompressed brush in thermal equilibrium $P=0$, of course. For very small compression, $1-D / h_{0} \leq 0.005$, the pressure is almost immeasurably small. Note, however, that there is some arbitrariness in defining $h_{0}$ exactly: here we have defined $h_{0}$ from the condition that $\phi(z)\{$ Fig. 2 h $\}$ has decreased to about $1 \%$ of its value in the flat region of $\phi(z)$ where the oscillations of $\phi(z)$ have just decayed. Note that the definition in terms of the first moment $\langle z\rangle$ of the density profile, $h_{0}=8\langle z\rangle / 3$, that is often used for flexible brushes and holds for the parabolic profile of the self-consistent field theory [1, 10, 11], is not useful for rather dense semi-flexible brushes, and hence not used here.
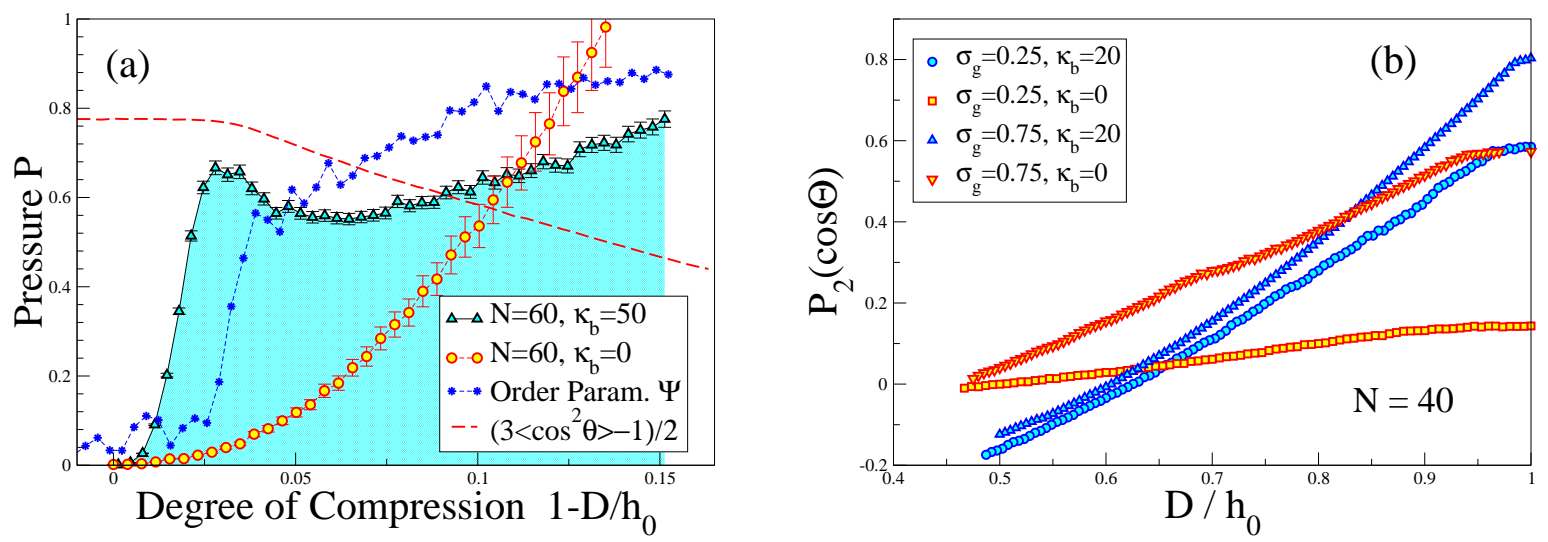

FIG. 7: (a) Normal pressure $\sigma P / k_{B} T$ for compressed brushes with chain length $N=60$ and grafting density $\sigma_{g}=0.5$ plotted vs. the degree of compression, $1-D / h_{0}$. The brush contains $N_{c h}=512$ chains. Both a flexible brush $\left(\kappa_{b}=0\right)$ and a brush formed from rather stiff chains $\left(\kappa_{b}=50\right)$ are included; for the latter also the order parameter $\Psi\{$ Eq 10\} and the orientational order parameter $P_{2}(\cos \theta)\{$ Eq. 12 $\}$ are shown, as indicated. (b) $P_{2}(\cos \theta)$ plotted vs. $D / h_{0}$ for the case $N=40$. Two values of the grafting density are shown $\left(\sigma_{g}=0.25\right.$ and 0.75 , respectively) for both flexible $\left(\kappa_{b}=0\right)$ and semi-flexible $\left(\kappa_{b}=20\right)$ chains.

While for flexible brushes, $\left(\kappa_{b}=0\right)$, the increase of the pressure is completely gradual for the full range of compressions, and can be described by a power law (roughly $P \propto D^{-3}$ for $\sigma \ll D \ll h_{0}$, as will be discussed below), the variation of the pressure for stiff chains is very different: there occurs a much faster rise up to a maximum, then 
the pressure decreases again to a shallow minimum, before a slow increase of the pressure occurs again. At large compressions the pressure in semi-flexible brushes clearly is distinctly smaller than in their flexible counterparts! The pressure maximum is due to the onset of collective orientational ordering of the chains in a particular direction in the $x y$-plane. A convenient way to measure this ordering in terms of the unit vectors $\vec{u}_{k}$ of the projections of the last bond vector of each chain into the $x y$-plane is

$$
\Psi=\left\langle\frac{1}{N_{c h}} \sqrt{\sum_{k=1}^{N_{c h}} u_{k, x}^{2}+\sum_{k=1}^{N_{c h}} u_{k, y}^{2}}\right\rangle
$$

as illustrated by the snapshots in Fig. 8 8 , b.
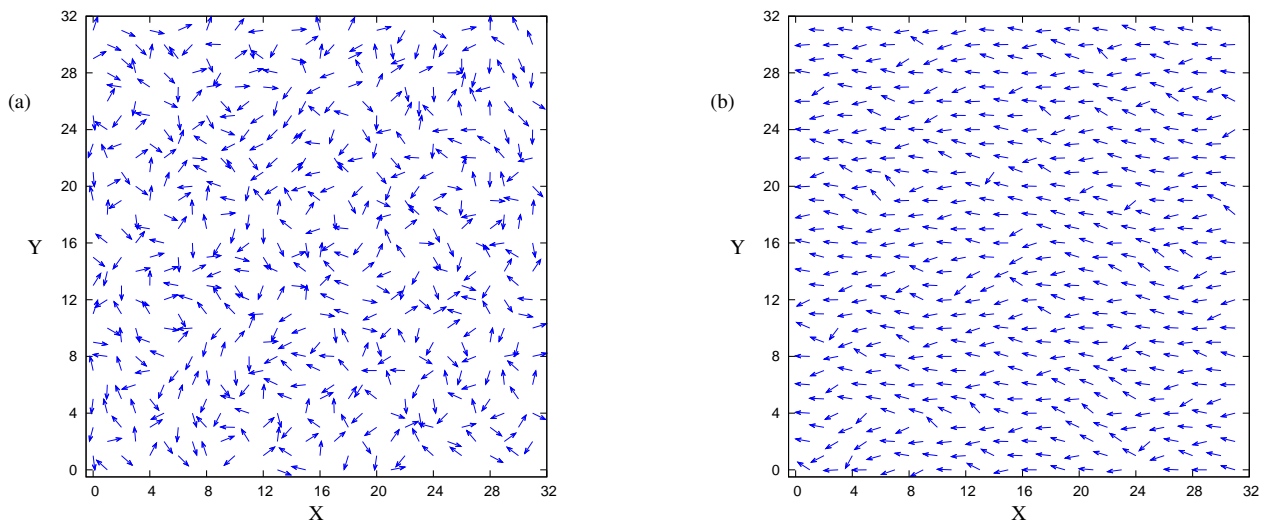

FIG. 8: Orientation of the last bonds in a polymer brush, represented by unit vectors in the $x, y$-plane for different degrees of compression $1-D / h_{0}$ : (a) no compression, orientational disorder; (b) $25 \%$ compression, orientational order. Here $N=$ 40, $\sigma_{g}=0.5, \kappa_{b}=50$, and the system size is $L \times L=32^{2}$ with $N_{c h}=512$.

If these unit vectors $\vec{u}_{k}$ are randomly oriented in the $x y$-plane, we obviously have $\Psi=1 / \sqrt{N_{c h}}$, and this is what is observed for zero (or very small) compression. This nonzero-plateau of $\Psi$ at zero compression is hence just a trivial finite size effect. On the other hand, if spontaneous symmetry-breaking occurs, and all unit vectors are parallel to each other, we would have $\Psi=1$. Such definitions of root mean square order parameters are well known from simulation studies of phase transitions in isotropic ferromagnets [60]. This gradual onset of ordering also shows up in the average angle that bonds make with the $z$-direction. Thus Fig. 7 b includes also data on $P_{2}(\cos \theta)$ defined as usual by

$$
P_{2}(\cos \theta)=\frac{1}{2}\left(3\left\langle\cos ^{2} \theta\right\rangle-1\right)
$$

While in the initial period of compression, up to the pressure maximum, $P_{2}(\cos \theta)$ stays essentially constant, then a gradual decline of $P_{2}(\cos \theta)$ sets in. Recall that $P_{2}(\cos \theta)=1$ for rods oriented along the $z$-axis while $P_{2}(\cos \theta)=-\frac{1}{2}$ for rods oriented parallel to the $x y$-plane. However, this smooth behavior of the order parameters $\Psi$ and $P_{2}(\cos \theta)$, indicative of a continuous (second-order) phase transition, is at variance with the observation of a loop (hysteresis) of the pressure in its variation with the compression. Such loops normally would be associated with a discontinuous (first order) phase transition. But a more careful consideration of equilibration reveals, (Fig. 9), that although some hysteresis between runs, where the compression is stepwise increased or decreased, is actually observed, it must be interpreted as an observation time effect: the amount of the hysteresis is almost completely gone, if the observation times are chosen 25 times larger! Of course, we expect that close to a second order transition relaxation times in the system become very long, due to "critical slowing down" 60, 61]. Thus, hysteresis is also known to occur for simulations of second order transitions [60], if the observation times at the individual state points near the transition are chosen too short. While for most systems this problem is not important in practice [60], here the situation differs because densely arranged stiff polymers are slow objects: the $x y$-like order of the last bond must be shared by a related order of the inner bonds of each chain as well. Figs. 7,9 focus on the behavior in the vicinity of the phase transition only.

It turns out, (Fig. 7b), that with strong compression the orientation of the bonds, which in Figs. 7.9 is still mostly along the $z$-direction, can be changed to a preference of $x, y$ direction: then, $P_{2}(\cos \theta)<0$ for semi-flexible brushes. In 


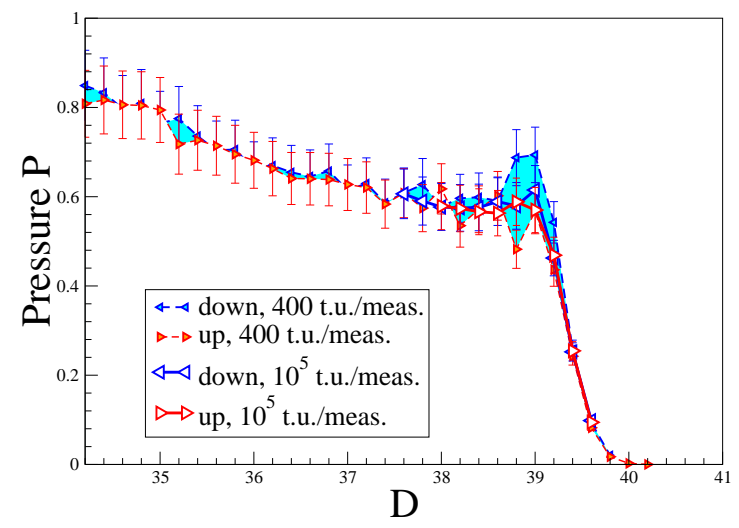

FIG. 9: Normal pressure $\sigma P / k_{B} T$ plotted vs. $D$ for the case $N=40, \kappa_{b}=50$ and $\sigma_{g}=0.50$, comparing runs where the pressure is stepwise increased and decreased again. In a short run, at each value of $D$ the system is held $400 \mathrm{MD}$ time units whereas in a long run, the equilibration time at every state was 25 times longer. The variance of measured values is also plotted as error bars.

contrast, flexible brushes at similar compressions, $\left(D / h_{0} \approx 0.5\right)$, would exhibit a rather random orientation of bond vectors, $P_{2}(\cos \theta) \approx 0$.

It is interesting to investigate this transition between a weakly compressed semi-flexible polymer brush (where $\Psi=0$ ), and the "buckling deformation" of individual stiff chains [33, 36, 41, 62]). One should bear in mind that this transition depends on the various parameters of the problem: bending stiffness $\kappa_{b}$, grafting density $\sigma_{g}$, and chain length $N$.
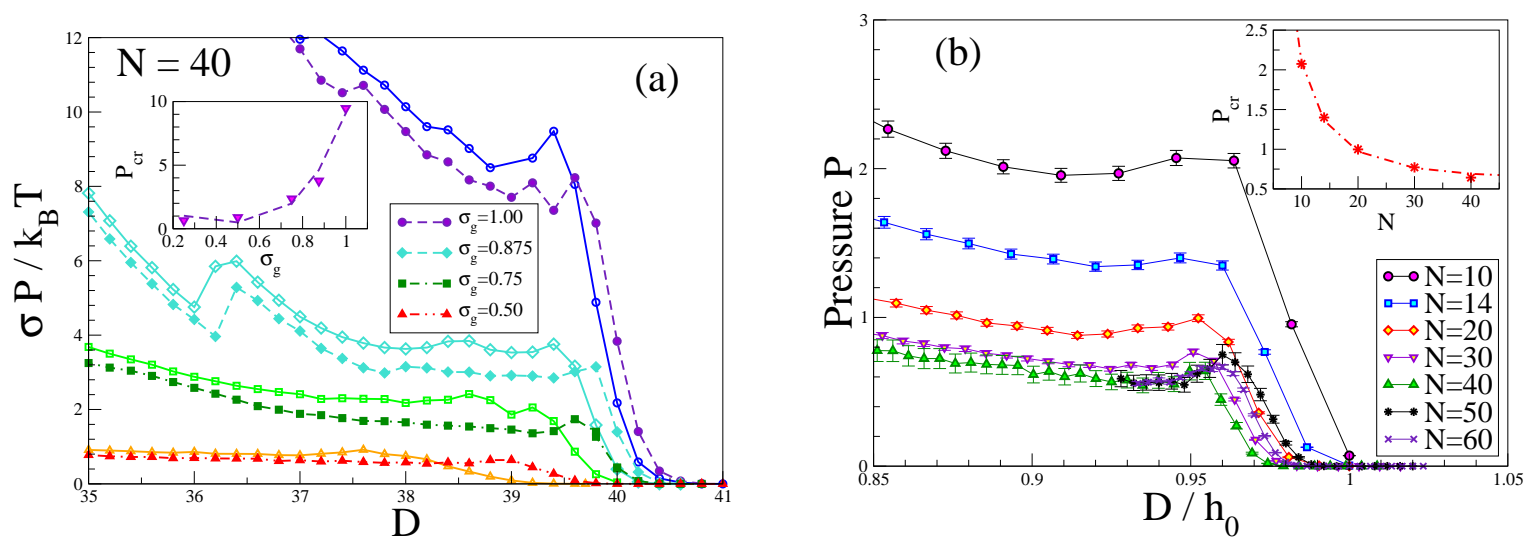

FIG. 10: (a) Normal pressure $\sigma P / k_{B} T$ plotted vs. distance $D$ for four choices of $\sigma_{g}$ and two choices of $\kappa_{b}, \kappa_{b}=20$ (empty symbols) and $\kappa_{b}=50$ (full symbols). All data are for $N=40$. The inset shows the variation of the critical pressure $P_{c r}$ with $\sigma_{g}$ for $\kappa_{b}=20$. Dashed line denotes a fit $P_{c r}=a_{1} \sigma_{g}+a_{2} \sigma_{g}^{2}+a_{3} \sigma_{g}^{3}$. (b) Normal pressure $\sigma P / k_{B} T$ plotted vs. $D / h_{0}$ for the case $\kappa_{b}=50, \sigma_{g}=0.5$, and a number of chain lengths from $N=10$ to $N=60$, as indicated. The inset shows $P_{c r}$ vs chain length $N$, dashed line is an empirical fit with $P_{c r}=c_{1} N^{-2}+c_{2}$.

Fig. 10 presents a selection of our data on this issue. One can see that the onset of compression results in a steeper $\sigma P / k_{B} T$ curve, when $\sigma_{g}$ increases (Fig. $7 \mathrm{~b}$ ), and also the almost flat plateau region of $\sigma P / k_{B} T$, that is reached at compressions beyond the maximum, rises with $\sigma_{g}$ very distinctly. The variation with $\kappa_{b}$ is rather weak, however: the primary effect is a slight increase of the brush height $h_{0}$ with $\kappa_{b}$, while the height of the flat region in the pressure gets slightly smaller with increasing $\kappa_{b}$ (presumably the chains order the better the stiffer they become). Moreover, the onset of bending occurs at smaller degree of compression, the denser the brush, $\sigma_{g}$, is. An important detail in this picture are also the observed occasional abrupt slides of the brush chains, as in the $P-D$ curves for $\sigma_{g}=0.875$ at $D \approx 36$, whereby some excess elastic energy stored in the system is released. It is conceivable that this effect is due to the finite (nonzero) rate of brush compression in the course of the MD simulation.

A very pronounced effect is seen when the chain lengths is varied, however (Fig. 10b): Very stiff short chains 
exhibit much larger pressures for comparable compressions $D / h_{0}$. Note that chains with $N=10, \kappa_{b}=50$ are in the limit where the persistence length $\ell_{p}$ (which equals $\kappa_{b}$ for our choice of units) exceeds by far the contour length $\mathcal{L} \approx 0.96(N-1)$, while for the chains with length $N=40$ to 60 both lengths are comparable. Interestingly, the data shown in Fig. 10b, imply that the pressure, when plotted versus $D / h_{0}$ for $D / h_{0}<0.95$ (i.e., after the onset of the $x y$-like ordering where chains bend uniformly in a chosen direction), depends on the persistence length only, and not on the chain length (while $h_{0}$ does depend strongly on $N$, as shown already in Fig. 2). The insets in Fig. 10 present the variation of the critical pressure $P_{c r}$ with grafting density. $P_{c r}$ itself is defined as the pressure $P$ where the second-order transition, manifested by the onset of lateral orientation order (in the $x y$-plane), takes place. For simplicity, we have estimated it roughly from the pressure maximum (a more precise study of this transition, e.g., by finite-size scaling methods [60], must be left to further work).

An important objection that could be raised against our findings is the suspicion that the regular square-lattice arrangement of our grafting sites leads to a structure of a dense semi-flexible brush that is much more regular than a real brush, for which the grafting sites are distributed at random. To check this caveat, we also performed a simulation for a typical choice of parameters $\left(N=40, \sigma_{g}=0.5, \kappa_{b}=50\right)$, where we compared the pressure variation with compression for two brushes, one with an ordered arrangement of grafting sites, and another one with an (almost) random grafting - Fig. 11. By "almost" random, we mean that the randomly chosen grafting sites were abandoned, if they were closer than a distance $\sigma$ to a grafting site that was already present (so as to account for the fact that the chemical groups that effect the grafting for two different chains are subject to excluded-volume effective repulsion).

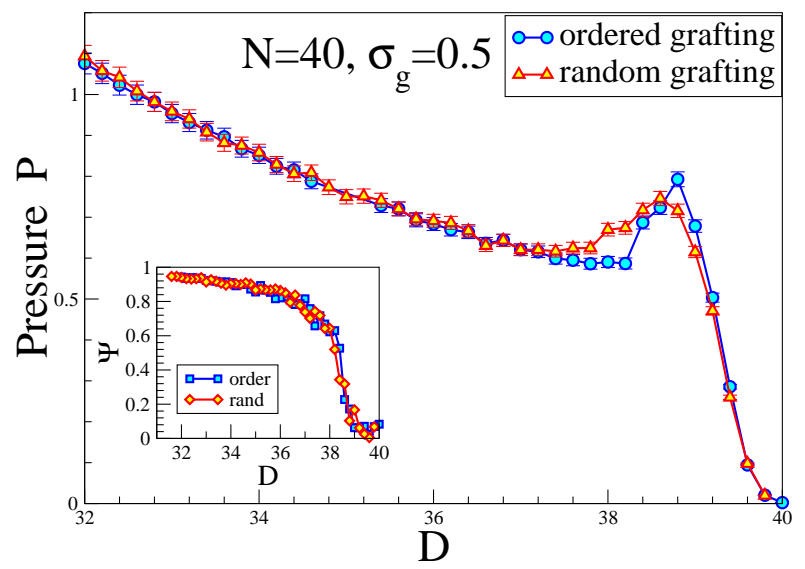

FIG. 11: A comparison of polymer brushes with ordered (circles) and random (triangles) grafting in their response to external pressure. Here the brush comprises 512 chains with $N=40$ and $\sigma_{g}=0.50$. The inset displays variation of the respective order parameter $\Psi$ with height $D$ of the compressing flat piston.

One sees that the pressure variation with piston height $D$ for the random choice of grafting sites hardly differs from that for regular arrangement except when the onset of order starts. In this narrow interval the $P-D$-relationship in the case of random grafting is slightly less sharp but rather smeared over a slightly broader interval of heights $D$, presumably indicating an effective "dilution" of the collective response due to randomness.

\section{A THEORETICAL DESCRIPTION}

We start with the observation that for grafting densities $\sigma_{g}>0.5$ and stiff chains $\left(\kappa_{b}>20\right)$, the polymers in the uncompressed state (for our choice of rather small chain length, $N<100$ ) are stretched out to their maximal length, that is, a rod-like conformation prevails. Transverse fluctuations are very small, as the $x y$-components of the gyration radius show - cf. Fig. [ Entropy plays then relatively little role and the equilibrium properties of a compressed brush in this limit should follow from a simple mechanical description.

The bending energy of a chain (including the uncompressed case) can be approximated as

$$
U_{\text {bend }}=\sum_{i=1}^{N-1}\left\langle V_{b}\left(\vartheta_{i-1, i, i+1}\right)\right\rangle \approx \frac{\kappa_{b}}{2} \sum_{i=1}^{N-1}\left\langle\vartheta_{i-1, i, i+1}^{2}\right\rangle \approx \frac{\kappa_{b}}{2} N\left\langle\vartheta^{2}\right\rangle
$$


In the last step one assumes that the distribution of bending energy along the backbone of the chain is approximately uniform. Fig. 12 supports this conclusion, and also implies the very interesting result that $\left\langle\vartheta_{i-1, i, i+1}\right\rangle \approx\langle\vartheta\rangle$ is
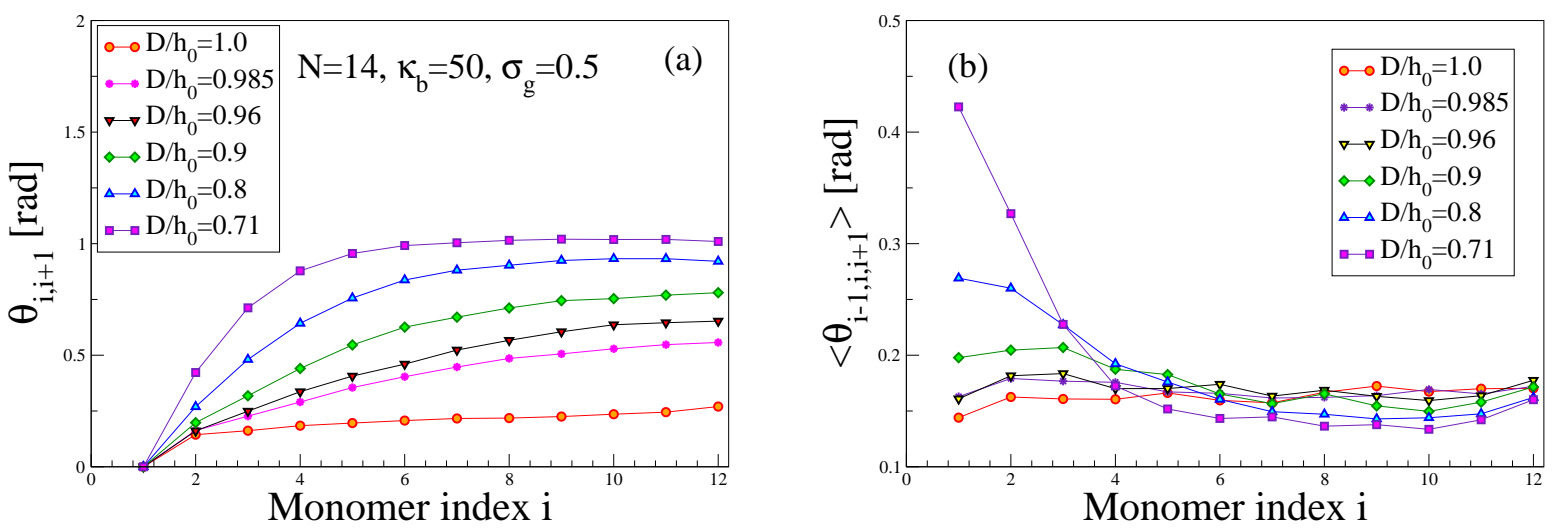

FIG. 12: (a) Variation of the angle $\theta_{i, i+1}$ of consecutive bonds along the chain backbone with the normal to the grafting surface at different degrees of compression $D / h_{0}$, as indicated. Here $N=14, \sigma_{g}=0.5$, and $\kappa_{b}=50$. (b) The same as in (a) for the angle $\vartheta_{i-1, i, i+1}$ between neighboring bonds.

independent of the compression $D / h_{0}$, at least for small deformations $D / h_{0} \geq 0.8$. Recall from Fig. 10 b that for this choice of parameters the pressure plateau (associated with the onset of lateral orientational order) starts at $D / h_{0} \approx 0.96$.

At zero pressure, and neglecting any interaction among the chains, one would find (recall that for $P=0$ the azimuthal angle $\varphi$ is still random and uniformly distributed, $\vartheta$ is a polar angle, and $\sin \vartheta \approx \vartheta$ for $\kappa_{b} \gg 1$ can be used)

$$
\langle\vartheta\rangle \approx \int_{0}^{\infty} \vartheta^{2} \exp \left(-\frac{\kappa_{b}}{2} \vartheta^{2}\right) d \vartheta / \int_{0}^{\infty} \vartheta \exp \left(-\frac{\kappa_{b}}{2} \vartheta^{2}\right) d \vartheta=\sqrt{\frac{\pi}{2 \kappa_{b}}}
$$

For the example shown in Fig. 12b we would obtain $\langle\vartheta\rangle \approx 0.17$, the value that is actually observed is close to this value at $D / h_{0}=1$. With increasing compression, larger angles for smaller $i$ are observed, i.e., the rod-like chains undergo strong bend in the vicinity of the substrate while staying straight away of this local deformation.

The opposite limit that we are considering is the case of perfect orientational ordering. Then the azimuthal angle $\varphi_{i, i+1}$ of all bonds from monomer $i$ to monomer $i+1$ remains the same for all bonds of all chains, and the polar angles $\theta_{i, i+1}$ of the bonds with respect to the $z$-axis lie in a plane characterized by this azimuthal angle $\varphi$ and containing the $z$-axis. In this limit a plausible assumption is that fluctuations of $\vartheta_{i-1, i, i+1}$ relative to their mean value $\langle\vartheta\rangle$ are small. Therefore we would conclude that

$$
U_{\text {bend }} \approx \frac{\kappa_{b}}{2} N\langle\vartheta\rangle^{2},\left\langle\theta_{i, i+1}\right\rangle=i\langle\vartheta\rangle, i=1,2, \ldots, N-1
$$

This result follows from the assumption that $U_{\text {bend }}$ has a minimum, subject to the constraint that in the case of perfect azimuthal ordering the last angle $\theta_{N-1, N}=\theta_{\max }$. Then one simply has to minimize the function $\tilde{U}=$ $U_{\text {bend }}-\lambda \sum_{i=1}^{N-1} \vartheta_{i-1, i, i+1}$ with respect to all the $\vartheta_{i-1, i, i+1}$ with $\lambda$ being a Lagrange multiplier. In this case one could derive a simple relation between $\langle\vartheta\rangle$ and the distance $D$ of the topmost monomer from the surface, as far as $\cos \left\langle\theta_{0,1}\right\rangle=1$,

$$
D=r_{\min } \sum_{i=0}^{N-1} \cos \left\langle\theta_{i, i+1}\right\rangle \approx r_{\min } \int_{0}^{N} \cos (s\langle\vartheta\rangle) d s=r_{\min } \frac{\sin (N\langle\vartheta\rangle)}{\langle\vartheta\rangle}
$$

Ignoring all other interactions among monomers, one then finds for the force $F$ exerted by a chain in the brush on the compressing plane

$$
F=\frac{\partial U_{\text {bend }}}{\partial D}=\frac{\partial U_{\text {bend }}}{\partial\langle\vartheta\rangle} \frac{\partial\langle\vartheta\rangle}{\partial D}=\frac{\kappa_{b}}{r_{\min }} \frac{\langle\vartheta\rangle^{2}}{\cos (N\langle\vartheta\rangle)-\frac{\sin (N\langle\vartheta\rangle)}{N\langle\vartheta\rangle}}
$$


The pressure $P$ would then be obtained simply by multiplying with the grafting density $\sigma_{g}$, to yield (for small $\langle\vartheta\rangle$ ) the result

$$
P=3 \sigma_{g} \frac{\kappa_{b}}{r_{\min } N^{2}}\left[1+\frac{1}{10}\langle\vartheta\rangle^{2}+\ldots\right]
$$

while $1-D / h_{0} \approx \frac{1}{6}(N\langle\vartheta\rangle)^{2}$ and $P_{2}(\cos \langle\theta\rangle) \approx 1-\frac{1}{2}(N\langle\vartheta\rangle)^{2}$.

Our simulation data, however, imply that such a description (intended to hold for the limit $\kappa_{b} \rightarrow \infty$ ) still lacks an important ingredient which we identify as the repulsive potential energy between the monomers. This energy clearly increases when we compress the system (simply due to density increase). Writing the volume fraction, occupied by the monomers, as $\phi=N r_{m i n}^{3} \sigma_{g} / D$, whereby $r_{\min }^{3}$ is taken as an estimate of the volume of a monomer, a mean-field estimate of the repulsive energy in the rod-like regime that prevails for $P<P_{c r}$ yields:

$$
U_{\text {rep }}=v \phi^{2} r_{\text {min }}^{2} D=v \frac{N^{2} \sigma_{g}^{2} r_{\min }^{8}}{D}=v \frac{h_{0}^{2} \sigma_{g}^{2} r_{\min }^{6}}{D}
$$

where $v$ is a constant (proportional to the second virial coefficient). The corresponding contribution to the pressure, $-\frac{\partial U_{r e p}}{r_{m i n}^{2} \partial D}=v\left(\frac{h_{0}}{D}\right)^{2} \sigma_{g}^{2} r_{m i n}^{4}$, has to be added to Eq. (18) and then a revised prediction for the critical pressure follows

$$
P_{c r}=\frac{3 \sigma_{g} \kappa_{b}}{r_{\min }} \frac{1}{N^{2}}+v \sigma_{g}^{2} r_{\min }^{4}
$$

Fig. $12 \mathrm{a}$ indicates, that the result $\left\langle\vartheta_{i-1, i, i+1}\right\rangle=i\langle\vartheta\rangle$ holds only for very small degrees of compression whereas for stronger compression, $D / h_{0} \leq 0.9$, it does not comply with our data. Snapshots of brush configurations - Fig. [13manifest instead that the polar angle $\theta_{i, i+1}$ attains its ultimate value $\theta_{\max }$ in the narrow interval encompassing the first few bonds only. Beyond this region of strong deformation in the vicinity of the grafting surface, the compressed semirigid polymer brush organizes itself into a quasi-crystalline nematic order of parallel bonds with constant inclination angle $\theta_{\max }$.
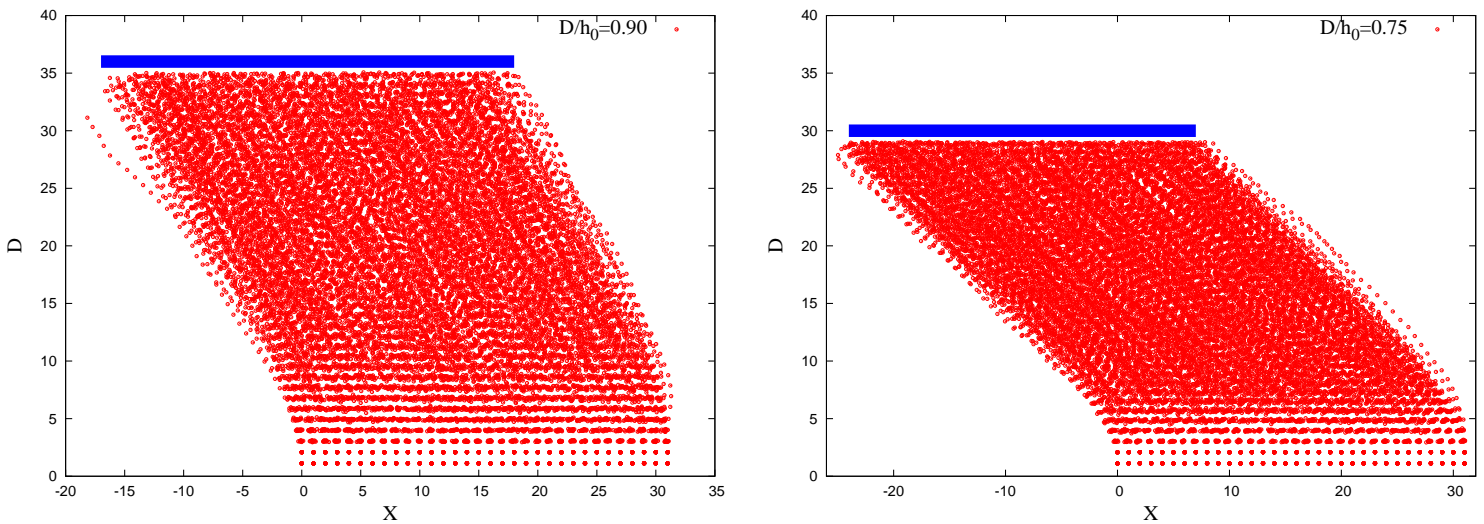

FIG. 13: Side view snapshots of a polymer brush with $N=40, \kappa_{b}=50$ at $\sigma_{g}=0.50$ and two heights $D$ of the upper wall (indicated in blue). (left) Degree of compression $D / h_{0}=0.90$, (right) $D / h_{0}=0.75$. Evidently, with increasing compression the contour of the polymer chains in the semi-rigid brush develops a "knickpoint" close to the grafting surface, rather than a regular arc that spans the two surfaces.

Eq. (20) was the motivation for the fit, used in the inset of Fig. 10p, in agreement with simulation measurements. In the inset of Fig. 10 a, we found it useful to include a term that could be attributed to the third virial coefficient and turn relevant in the limit of very large grafting density $\sigma_{g} \rightarrow 1$.

In what follows we replace the discrete index $i$ of each monomer by a continuous variable: $\theta_{i, i+1} \rightarrow \theta(s)$. From Figs. 12 , 13 we recognize that the bond orientations $\theta(s)$ along the chain contour, cf. Eq. 16, describes a nontrivial curve in the plane, singled out by the symmetry breaking of the azimuthal angle $\varphi$ due to orientational order. If one would only take the bending energy into account, one would obtain the trivial linear variation $\theta(s)=s\langle\theta\rangle$ of Eq. 15. In order to derive a reasonable approximation for $\theta(s)$, it is necessary to take into account both the energy 
due to compression, that is, a 'pressure times volume' term, which is $U_{\text {comp }}(\theta)=P \sigma_{g} r_{\text {min }}^{5} \int_{0}^{N} d s \cos \theta(s)$, as well as the energy of monomer - monomer repulsion, $U_{\text {rep }}(\theta)$. Indeed, with growing $P$, as the stiff chains bend more and more, the monomers get closer to one another, unlike the case when the chains are vertically stretched out like stiff rods. A simple geometric argument, cf. Fig. 14a, yields thus

$$
U_{r e p}(\theta) \equiv v \sigma_{g}^{2} r_{\min }^{7} \int_{0}^{N} \frac{d s}{\cos \theta(s)}
$$

For $\theta(s)=0$, Eq. (21) leads back to Eq. (19), of course. Putting all terms together, the free energy that needs to be minimized, becomes (using the abbreviations $P^{\prime}=P \sigma_{g} r_{\text {min }}^{5}, v^{\prime}=v \sigma_{g}^{2} r_{\text {min }}^{7}$ ) becomes since the normal distance between rods in an array of rods, tilted by angle $\theta$ scale like $\cos \theta^{-1}$ with respect to the $z$-axis.

$$
F=\int_{0}^{N} d s\left[\frac{\kappa_{b} \sigma_{g}}{2}\left(\frac{d \theta}{d s}\right)^{2}+\frac{v^{\prime}}{\cos \theta(s)}+P^{\prime} \cos \theta(s)\right]
$$

which yields upon minimization with respect to $\theta(s)$ the following Euler - Lagrange equation:

$$
\kappa_{b} \sigma_{g} \frac{d^{2} \theta}{d s^{2}}-v^{\prime} \frac{\sin \theta(s)}{\cos ^{2} \theta(s)}+P^{\prime} \sin \theta(s)=0 .
$$

In the region where $\theta(s)$ is still small, one can expand Eq. (23) keeping only the terms up to order $\theta^{3}$, arriving at

$$
\frac{d^{2} \theta}{d s^{2}}+m^{2} \theta(s)-w^{2} \theta(s)^{3}=0
$$

where the abbreviations

$$
m^{2} \equiv \frac{P^{\prime}-v^{\prime}}{\kappa_{b} \sigma_{g}}, w^{2} \equiv \frac{P^{\prime}+5 v^{\prime}}{6 \kappa_{b} \sigma_{g}}
$$

have been introduced. Note that considerable deviations of $\theta$ from zero, and the ensuing increased importance of the repulsion energy, Eq. (21), emerge when the applied compressing pressure $P$ is sufficiently large so that the condition $P^{\prime}>v^{\prime}$, i.e., $m^{2}>0$ in Eq. (25) appears physically reasonable.
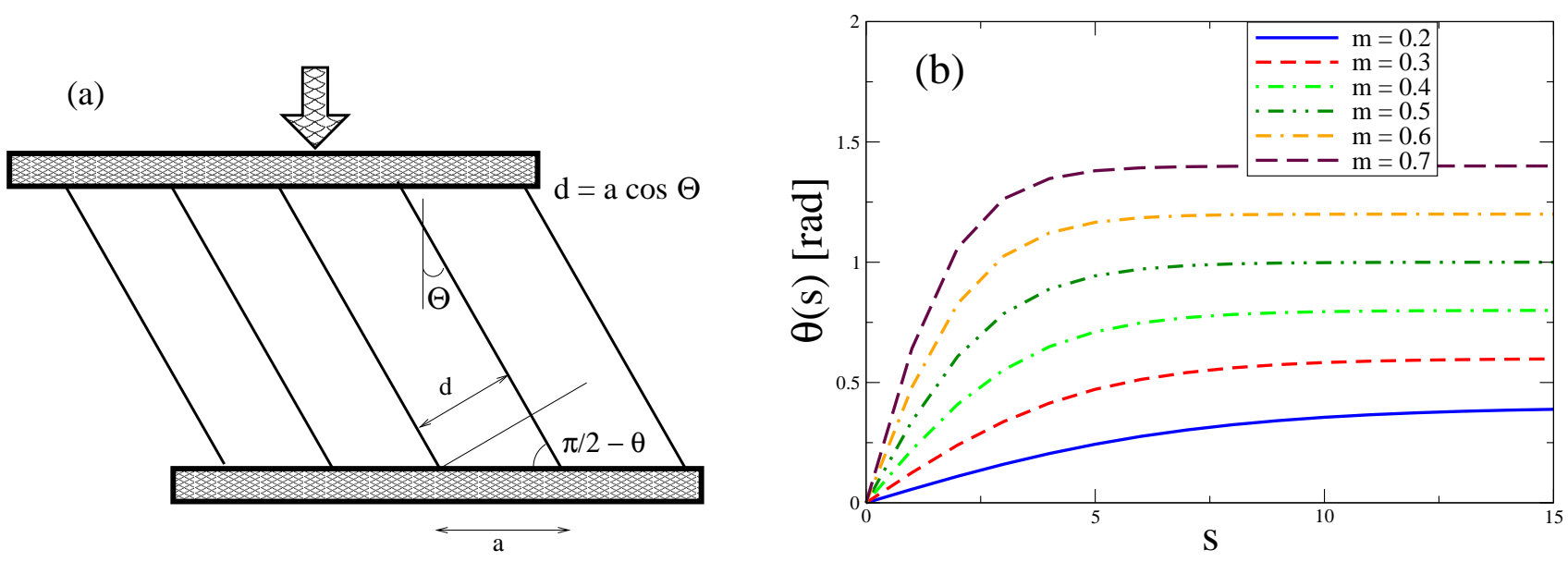

FIG. 14: (a) As the rod-like chains tilt at growing angle $\theta>0$, the distance $d$ between neighboring rods diminishes like $d=a \cos \theta$, where $a$ is the separation between grafting points. (b) Variation of the polar angle $\theta(s)=\frac{m}{w} \tanh \left(\frac{m}{\sqrt{2}} s\right)$ along the chain contour length of deformed polymer chains with $N=14$ beads, $w=0.5$, and different values of the parameter $m=\sqrt{\frac{P^{\prime}-v^{\prime}}{\kappa_{b} \sigma_{g}}}$, as indicated. Apparently, the bending increases with growing pressure $P^{\prime}$, and even more so for softer, $\kappa_{b} \rightarrow 0$, or less dense brushes. 
The approximation, involved in reducing Eq. (23) to Eq. (24), holds for not too large angles $\theta$ only, and also Eq. (22) can only hold at high grafting density $\sigma_{g}$ (otherwise the neglect of entropy and the assumption of perfect symmetry breaking of the azimuthal angle cannot be true). For sufficiently strong pressure, large angles $\theta>1$ occur, and one must then solve the full Eq. (23).

The governing Eq. (24), derived in the present treatment, is familiar from the so called $\phi^{4}$-model of statistical mechanics, and has emerged in different contexts. With respect to the boundary condition, $\theta(s \rightarrow 0)=0$, one obtains the well-known soliton (kink) solution

$$
\theta(s)=\frac{m}{w} \tanh \left(\frac{m}{\sqrt{2}} s\right)=\sqrt{\frac{P^{\prime}-v^{\prime}}{P^{\prime}+5 v^{\prime}}} \tanh \left(\sqrt{\frac{P^{\prime}-v^{\prime}}{2 \kappa_{b} \sigma_{g}}} s\right) .
$$

where $m$ and $w$ are positive constants, cf. Eq. (25). Indeed, Fig. 14 demonstrates that the solution, Eq. (26), nicely reproduces the observed MD data, shown in Fig. (12)a, at least qualitatively. Eventually, one should note that going back to the case of small deformation when $\theta \rightarrow 0$, and the energy contribution due to chain repulsion, Eq. (21), can be neglected, a solution of our governing equation, Eq. (23), recovers the expression for the critical pressure $P_{c r}$, namely, Eq. (20), as expected [24]. It is worth mentioning, that this result, Eq. (20), has been first obtain by L. Euler and D. Bernoulli for the famous 'beam bending' problem in the year 1750 .

\section{OUTLOOK ON DYNAMIC BEHAVIOR}

In polymer brushes one may study relaxation phenomena on many different levels: (i) fluctuations of individual monomers and and motion of chains as a whole in thermal equilibrium 64], (ii) collective relaxation phenomena as described by dynamic correlations of the local monomer density in the brush, (iii) the nonlinear dynamic response, associated with compression and shear, e.g. [10]. A comprehensive treatment for any of these topics for brushes formed from rather stiff chain molecules is beyond the scope of the present paper yet here we present few results which relate to the relaxation (brush recovery), associated with changes of the compression $D / h_{0}$ of the brush (recall the hysteresis observed near the onset of lateral orientational ordering). A very straightforward computer experiment, which could also possibly carried out in the laboratory, considers the relaxation of a compressed brush after the compressing piston at time $t=0$ is suddenly removed and the brush relaxes from its height $h(t=0)=D$ back to its uncompressed state with height $h_{0}$. Fig. 15 shows the time dependence of the $z$ - and $x y$-components of both the end-to-end vector and gyration radius square for few typical cases of such computer experiment. The data are shown as semi-logarithmic plots which would lead to straight lines, if the relaxation functions plotted were simple exponential (which obviously is not the case). Fig. [15a indicates that the relaxation behavior of the $x y-$ and $z$-components is similar, and Fig. 15 p shows that the initial decay becomes much faster with increasing grafting density. Fig. 15 includes also an empirical fit to a function const. $t^{-1 / 2} \exp \left(-t / \tau_{R}\right)$ where the relaxation time $\tau_{R}$ is a second adjustable parameter, $\tau_{R} \approx 40$.

These measurements are compared to those concerning time recovery of an initially compressed brush comprised of flexible chains, $\kappa_{b}=0$, and otherwise identical parameters - Fig. 15k, d. Apparently, in the absence of chain stiffness the relaxation kinetics for the $z$ and $x y$-components differs significantly: components perpendicular to the grafting surface undergo short-termed fast recovery followed by a much slower secondary relaxation whereas the relaxation of the parallel components appears to be governed by a power law $\propto t^{-1 / 4}-$ Fig. 15 d.

\section{CONCLUSIONS}

In this work Molecular Dynamics simulations of dense polymer brushes, formed from semi-flexible chains were presented, focusing on the regime where persistence length and contour length of these rather stiff macromolecules are comparable. In addition, the chemical nature of the grafted first bond renders it perpendicular to the underlying surface and, therefore, shapes the overall brush behavior.

We show that this behavior is very different from what is known for brushes comprised of flexible chains. It turns out that the chains in the brush at high grafting density behave nearly as rigid rods, and the $z$-components of the chain end-to-end distance and gyration radius square take their maximal possible values, provided the chemistry of chain grafting fixes the first bond perpendicular to the substrate.

It is shown that fluctuations of the chain in the transverse $(x y)$-directions, perpendicular to the $z$-axis along which the rods are oriented, are extremely small for large bending stiffness, and decrease like a simple exponential with growing grafting density $\sigma_{g}$ - cf. Fig. 4 a . In contrast, the lateral size of a chains, $R_{g x y}^{2}, R_{x y}^{2}$, changes non-monotonically with stiffness and goes through a maximum for a finite degree of rigidity. This behavior is very different from what is known for flexible chains in brushes in the case of semi-dilute and concentrated monomer densities in the brush. 

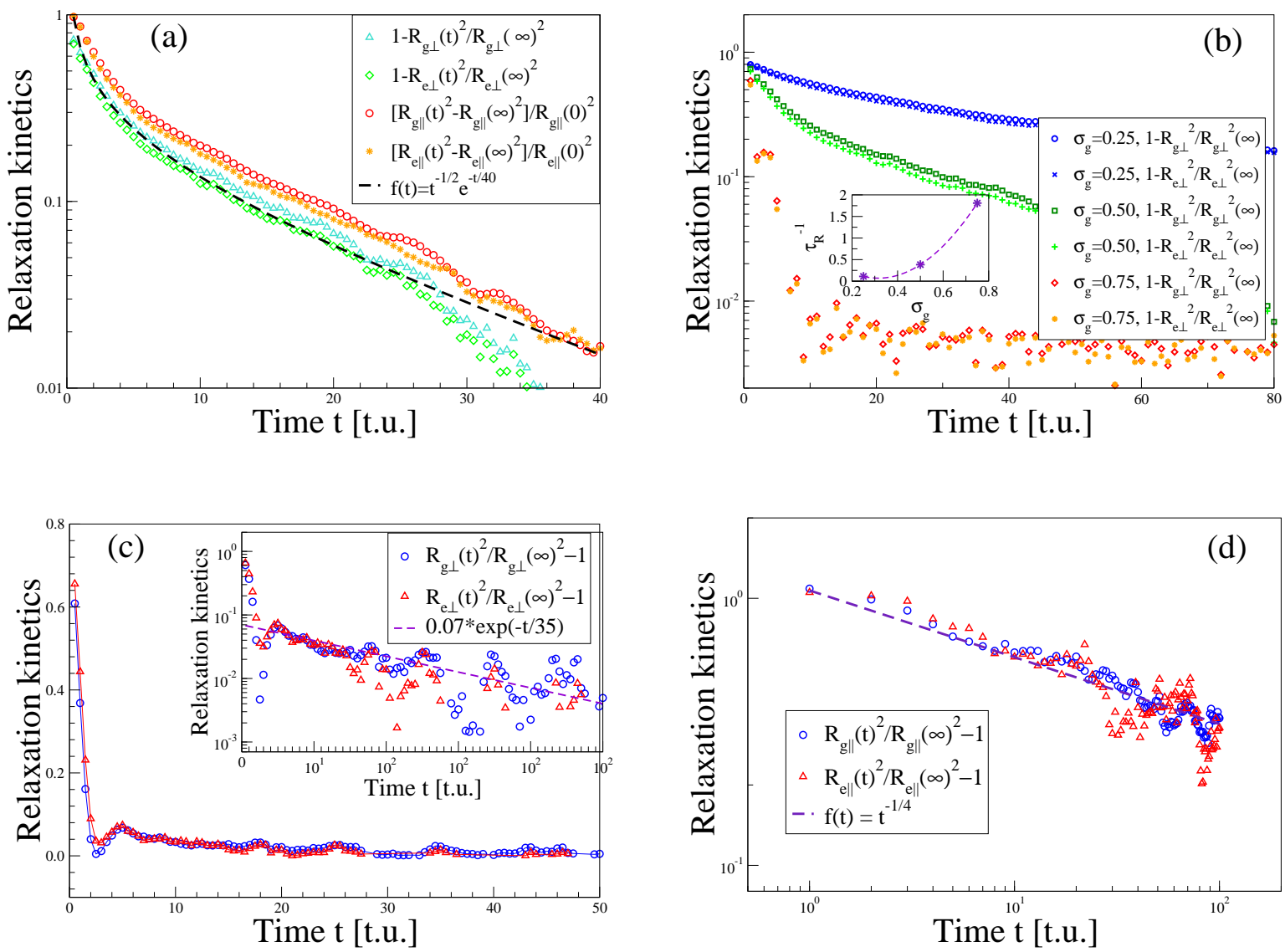

FIG. 15: Relaxation functions of perpendicular and parallel mean square components of the gyration radius of the chains. The polymer brush recovery was monitored after the compressing piston was instantaneously removed at time $t=0$. All cases shown refer to $N=40, \kappa_{b}=20$, and $D(t=0) / h_{0}=1 / 2$. Case (a) shows $\sigma_{g}=0.5$, while (b) compares three grafting densities, as indicated. The characteristic relaxation time $\tau_{R}$ for initial brush recovery is shown in the inset as function of $\sigma_{g}$ along with a fit by $\tau_{R}^{-1}=b_{0}+b_{1} \sigma_{g}+b_{2} \sigma_{g}^{2}$. A comparison with the recovery kinetics of a polymer brush at $\sigma_{g}=0.5$, comprised of totally flexible chains, $\kappa_{b}=0$, is presented in (c) and (d). Here again $N=40$.

Only for the case of melt densities, crystallization in polymer brushes has been predicted [63] which may be related to our observations.

In the present work we have focused on the compression of these stiff chain brushes and on the resulting orientational order of the bonds in the $x y$-directions during bending. We have demonstrated that the onset of this order is a continuous phase transition where the rotational symmetry in the $x y$-plane is broken. However, since all bonds of the chains in a perfectly ordered system would have to share this ordering in the simulation box, requiring large lateral monomer displacements in the same direction, there is obviously a commensurability conflict between the lateral deformation of such a brush and the periodic boundary conditions. This singles out the $x, y$ axes, imposing a cubic symmetry of the bending phenomenon. Due to these problems, a study of the critical behavior of this phase transition would be premature, and has not been attempted.

In order to obtain theoretical guidance on the observed phenomenon, some phenomenological considerations were presented. Again we emphasize that these considerations and the resulting interesting predictions, cf. Section V. should be taken as a first step only while a more systematic theory even on the mean-filed level remains a challenge for the future.

One interesting feature is the finding that for large compression the resilient pressure of such stiff chain brushes, exerted on the compressing piston, is much smaller that for flexible chain brushes of the same chain length and grafting density. Thus, a study of shear forces between two stiff chain brushes would also be very interesting but must be left as a future task too.

In conclusion, we hope that the present work will stimulate experimental interest in this subject. We also believe that some related phenomena may take place in dense arrangements of stiff biopolymers in the context of complex 
biological structures.

\section{ACKNOWLEDGMENTS}

We are indebted to Friederike Schmid, Oleg Borisov, and Burkhard Dünweg for instructive discussions of this work. This investigation was supported by the Deutsche Forschungsgemeinschaft (DFG) under Grant No Bi $314 / 23$.

[1] S. Milner, Science, 1991, 251, 905.

[2] A. Halperin, M. Tirrell and T. P. Lodge, Adv. Polym. Sci., 1992, 100, 31.

[3] G. S. Grest and M. Murat, in Monte Carlo and Molecular Dynamics Simulations in Polymer Science, ed. by K. Binder, p. 476, Oxford Univ. Press, New York, 1995.

[4] J. Klein, Annu. Revs. Mater. Sci., 1996, 26, 581.

[5] I. Szleifer and M. Carignano, Adv. Chem. Phys., 1996, 94, 165.

[6] G. S. Grest, Adv. Polym. Sci., 1999, 138, 149.

[7] L. Leger, E. Raphasel, and H. Hervet, Adv. Polym. Sci., 1999, 138, 185.

[8] R. C. Advincula, W. J. Brittain, K. C. Caster, and J. Rühe, (eds.) Polymer Brushes, Wiley-VCH, Weinheim, 2004.

[9] R. Descas, J.-U. Sommer, and A. Blumen, Macromolecular Theory Simul, 2008, 17, 429.

[10] K. Binder, T. Kreer, and A. Milchev, Soft Matter, 2011, 7, 7159.

[11] K. Binder and A. Milchev, J. Polym. Sci. B: Polym. Phys., 2012, 50, 1515.

[12] S. Alexander, J. Phys. (Paris), 1977, 38, 983.

[13] P. G. de Gennes, Macromolecules, 1980, 13, 1069.

[14] A. Yu. Grosberg and A. R. Khokhlov,Statistical Physics of Macromolecules, AIP Press, New York, 1994.

[15] M. Carignano and I. Szleifer, J. Chem. Phys., 1993, 98, 5006.

[16] G. G. Kim and K. Char, Bull. Korean Chem. Soc. 1999, 20, 1026.

[17] C.-M. Chen and Y.-A. Fwu, Phys. Rev. E, 2000, 63, 011506.

[18] A. Gopinath and I. Mahadevan, Proc. R. Soc. London Ser. A, 2011, 467, 1665.

[19] A. Milchev and K. Binder, J. Chem. Phys., 2012, 136, 104901.

[20] D. R. M. Williams and A. Halperin, Europhys. Lett. 1992, 19, 963.

[21] D. R. M. Williams and A. Halperin, Macromolecules, 1993, 28, 2025.

[22] A. Halperin and D. R. M. Williams, Europhys. Lett. 1993, 21, 575.

[23] D. R. M. Williams and A. Halperin, Macromolecules, 1993, 26, 4208.

[24] D. R. M. Williams and A. Halperin, Langmuir, 1995, 11, 3626.

[25] F. M. Haas, R. Hilfer, and K. Binder, J. Phys. Chem. 1996, 100, 15290.

[26] V. M. Kaganer, H. Möhwald, and P. Dutta, Rev. Mod. Phys. 1999, 71, 779.

[27] F. Schmid, D. Johannsmann, and A. Halperin, J. Phys. II (Paris), 1996, 6, 1331

[28] K. Kegler, M. Salomo, and F. Kremer, Phys. Rev. Lett. 2007, 98, 058304

[29] K. Kegler, M. Konieczny, G. Dominguez-Espinosa, C. Gutsche, M. Salomo, F. Kremer, and C. N. Likos2, Phys. Rev. Lett. 2008, 100, 118302

[30] G. Dominguez-Espinosa, A. Synytska, A. Drechsler, C. Gutsche, K. Kegler, P. Uhlmann, M. Stamm, F. Kremer, Polymer, 2008, 49, 4802

[31] G. Wong, A. Lin, J. Tang, Y. Li, P. Janmey, and C. Safinya, Phys. Rev. Lett., 2003, 91, 018103.

[32] J. H. Shin, M. L. Gardel, L. Mahadevan, P. Matsudaira, and D. Weitz, PNAS 2004, $101,0636$.

[33] J. Kierfeld, P. Gutjahr, T. Kühne, P. Kraikivski, and R. Lipowsky, J. Comput. Theor. Nanosci. $2006,3,898$.

[34] M. Bathe, C. Heussinger, M. M. A. E. Claessens, A. R. Bausch and E. Frey, Biophys. J. 2008, $94,2955$.

[35] O. Lieleg, K. M. Schmoller, C. J. Cyron, Y. Luan, W. A. Wall and A .R. Bausch, Soft Matter, $2009,5,1796$.

[36] J. Kierfeld, K. Baczynski, P. Gutjahr and R. Lipowsky, AIP Conf. Proc., 2008, 1002, 151.

[37] S. K. Lakkaraju and W. Hwang, Phys. Rev. Lett., 2009, 102, 118102.

[38] J. R. Blundell and E. M. Terentjew, Soft Matter, 2009, 5, 4015.

[39] Y. Higari, R. Okazaki, and A. Takahara, ACS Macro Lett., 2012, 1, 1124.

[40] T. Moro, Y. Takatori, K. Ishihara, T. Konno, Y. Takigawa, T. Matsushita, U. Chung, K. Nakamura, and H. Kawaguchi, Nat. Mater. 2004, 3, 829.

[41] J. Klein, Proc. IMech. E J, 2006, 220, 691.

[42] J. Klein, Science, 2009, 323, 47.

[43] S. T. Milner, T. A. Witten, and M. E. Cates, Macromolecules 1988, 21, 2610.

[44] S. T. Milner, Europhys. Lett. 1988, 7, 695.

[45] E. B. Zhulina, O. V. Borisov, and V. A. Prymatsyn, J. Coll. Sci. 1990, 137, 495.

[46] A. Galuschko, L. Spirin, T. Kreer, A. Johner, C. Pastorino, J. Wittmer, and J. Baschnagel, Langmuir, $2010,26,6418$.

[47] S. M. Balko, T. Kreer, P. J. Costanzo, T. E. Patten, A. Johner, T. L. Kuhl, and C. M. Margues, PLOS ONE, 2013, 8, Issue 3, e58392. 
[48] T. Kreer and S. M. Balko, ACS Macro Lett. 2013,

[49] A. Milchev and K. Binder, EPL, 2013, 102, 58003.

[50] M. Murat and G. S. Grest, Macromolecules 1989, 22, 4054.

[51] G. S. Grest and K. Kremer, Phys. Rev. A 1986, 33, 3028.

[52] J. D. Weeks, D. Chandler, H. C. Andersen, J. Chem. Phys., 1971, 54, 5237.

[53] O. Kratky and G. Porod, J. Colloid. Sci., 1949, 4, 35.

[54] V. A. Ivanov, A. S. Rodionova, J. A. Martemyanova, M. R. Stukan, M. Müller, W. Paul and K. Binder, Macromolecules, 2013, (submitted)

[55] H.-P. Hsu, W. Paul and K. Binder, Macromolecules, 2010, 43, 3094.

[56] M. P. Allen and D. J. Tildesley, Computer Simulation of Fluids (Clarendon Press, Oxford, 1987).

[57] I. K. Snook, The Langevin and Generalized LAngevin Approach of the Dynamics of Atomic, Polymeric, and Colloidal Systems (Elsevier, Amsterdam, 2007)

[58] T. Odijk, Macromolecules, 1983, 16, 1340

[59] H.-P. Hsu and K. Binder, J. Chem. Phys., 2012, 136, 024901.

[60] K. Binder and D. W. Heermann, Monte Carlo Simulation in Statistical Physics. An Introduction. 5th ed. (Springer, Berlin, 2010)

[61] P. C. Hohenberg and B. I. Halperin, Rev. Mod. Phys. 1977,

[62] J. R. Blundell and E. M. Terentjew, Soft Matter 2009, 5, 41015.

[63] Gui-Li He, Holger Merlitz, Jens-Uwe Sommer and Chen-Xu Wu, Eur. J. Phys. E 2007 24, 325

[64] D. Reith, A. Milchev, P. Virnau, and K. Binder, Macromolecules, 2012, 45, 4381

[65] G. Arya, J. Phys. Chem. B, 2010, 114, 15886.

[66] This kind of chemical grafting is referred to in the literature as constraint attachment [65] and shown to reveal quite unexpected effects of stiffness in the context of poly-electrolyte colloids. 
For Table of Contents Use Only

Unconventional ordering behavior of semi-flexible polymers in dense brushes under compression by

Andrey Milchev, and Kurt Binder
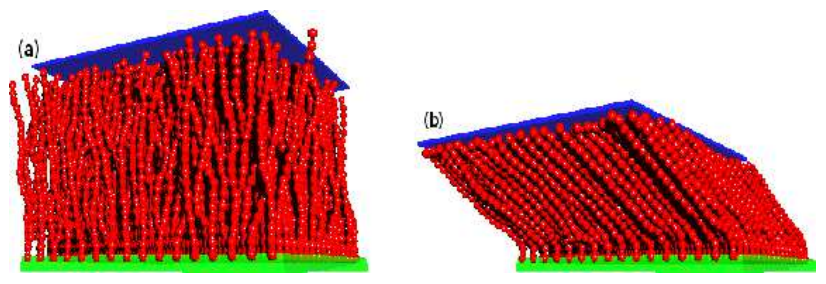

TOC graphics: Snapshots of a compressed semi-flexible brush under flat piston at two different degrees of deformation. 